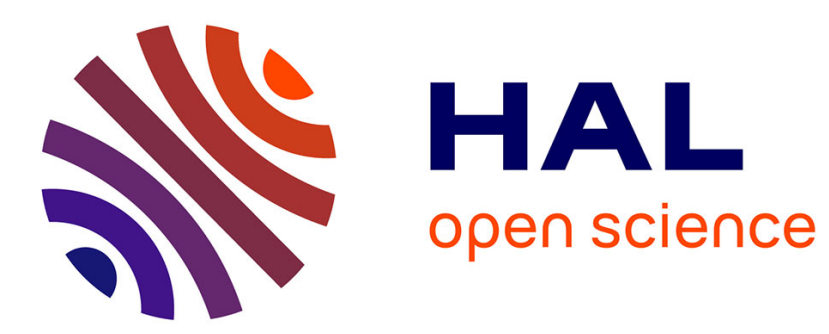

\title{
Transition to turbulence in open flows: what linear and fully nonlinear local and global theories tell us Jean-Marc Chomaz
}

\section{To cite this version:}

Jean-Marc Chomaz. Transition to turbulence in open flows: what linear and fully nonlinear local and global theories tell us. European Journal of Mechanics - B/Fluids, 2004, 23 (13), pp.385-399. 10.1016/j.euromechflu.2003.10.006 . hal-01025808

\section{HAL Id: hal-01025808 \\ https://hal-polytechnique.archives-ouvertes.fr/hal-01025808}

Submitted on 8 Dec 2017

HAL is a multi-disciplinary open access archive for the deposit and dissemination of scientific research documents, whether they are published or not. The documents may come from teaching and research institutions in France or abroad, or from public or private research centers.
L'archive ouverte pluridisciplinaire HAL, est destinée au dépôt et à la diffusion de documents scientifiques de niveau recherche, publiés ou non, émanant des établissements d'enseignement et de recherche français ou étrangers, des laboratoires publics ou privés. 


\title{
Transition to turbulence in open flows: what linear and fully nonlinear local and global theories tell us
}

\author{
Jean-Marc Chomaz
}

LadHyX - CNRS, École polytechnique, 91128 Palaiseau, France

\begin{abstract}
Mixing layers, jets, wakes, boundary layers over wings or rotating disks, Poiseuille and Couette flows are examples of open shear flows encountered in many industrial or geophysical situations. These flows develop spatially under the combined action of advection and instabilities and eventually undergo a transition to turbulence.

In the eighties, the linear concepts of absolute and convective instability succeeded in predicting some aspects of open shear flow dynamics, but a description of their spatio-temporal development including nonlinear effects and secondary instabilities was lacking and even the very fact that a linear criterion describes so well strongly nonlinear flows remains mysterious.

The present work reports on very recent progress elucidating open shear flow dynamics. A fully nonlinear extension of the concepts of absolute and convective instability introduced by Chomaz (Phys. Rev. Lett. 69 (1992) 1931) is recalled in connection with the broader problem of front and pattern selection. These new ideas are first illustrated on simple amplitude equations. Then the fully nonlinear concepts are applied to actual flows such as wakes and mixing layers. Furthermore, new scenarii involving secondary absolute instability are proposed and compared to the dynamics of the rotating disk and mixing layers experiment.
\end{abstract}

Keywords: Secondary instability; Wakes; Global modes; Mixing layers; Absolute/convective instability; Front

\section{Introduction}

When investigating the dynamics of a parallel mixing layer, it is well established (Strykowski and Niccum [1], Strykowski, Krothapalli and Jendoubi [2]) that, when the backflow is large enough, the primary instability becomes absolute. In that case a so-called Global Mode should appear and should lead to a regular shedding of vortices and to the formation of a single vortex street. But for the very value of the backflow at which the primary instability becomes absolute Brancher and Chomaz [3] showed, analyzing the Stuart model of a single vortex street, that, when formed, the street is already absolutely unstable to two-dimensional (pairing) and three-dimensional (translative) instabilities (see also Brancher [4]). The case, when the absolute secondary instability precedes the primary one, constitutes a new generic scenario for a one step bifurcation to disorder referred to as the AA route, already discussed by Couairon and Chomaz [5] when analysing model problems. The corresponding dynamics is illustrated here on a numerical simulation of a parallel mixing layer. Its occurrence for hot or forced jets, flows over a backward facing step or over a rotating disk might explain how these flows experience a transition to turbulence. 


\section{Nonlinear absolute instability}

It is well understood that an open flow may be globally stable although it is locally unstable, i.e., unstable if the local velocity profile is extended to infinity in the streamwise direction (see Huerre and Monkewitz [6]). This is simply because, the perturbations are constantly transported away from the unstable region. While an initial compact perturbation is travelling in the locally unstable region it grows but when it leaves the unstable region it decays. The mean flow has a global stabilizing effect. As a result, globally stable open flows exhibit large transient growth and large inlet noise amplification but at large time and in the absence of external forcing they relax back to their basic flow. This behaviour correspond from a local point of view to the concept of convective instability and from the global point of view to the fact that the global linearized evolution operator is strongly nonnormal (see Cossu and Chomaz [7], for details). When a resonance occurs, the local amplification of perturbation is already very intense and the flow becomes rapidly fully nonlinear. One would therefore imagine that a fully nonlinear criterion should be invoked to explain transition. Surprisingly this is in general not the case and linear absolute instability has been able to predict the occurrence of self-sustained resonance in counter-flow mixing layers (Strykowski and Niccum [1], Strykowski, Krothapalli and Jendoubi [2]), wakes with or without suction (Hammond and Redekopp [8], Woodley and Peake [9], Leu and Ho [10]), hot or helium jets (Sreenivasan et al. [11], Monkewitz et al. [12], Kyle and Sreenivasan [13], Yu and Monkewitz [14]). For the rotating disk the absolute instability threshold is associated with transition to turbulence instead of regular oscillation (Lingwood [15-18]). This may be typical of the AA route discussed in the present paper. Recently, the analysis of model equations describing the evolution of instability waves demonstrated how a linear criterion may define the occurrence of a strongly nonlinear self-sustained oscillation. The basic idea was proposed in Chomaz [19] where the nonlinear concept of absolute and convective instabilities was introduced (Fig. 1): "The basic state of a system is nonlinearly stable (NS) if for all initial perturbations of finite extent and amplitude, the system relaxes to the basic state everywhere in any moving frame. The system is unstable if it is not stable in the above sense. The instability is nonlinearly convective (NC) if, for all initial perturbations of finite extent and finite amplitude, the system relaxes to the basic state everywhere in the laboratory frame. It is nonlinearly absolute (NA) if, for some initial condition of finite extent and amplitude, the system does not relax to the basic state everywhere in the laboratory frame." Here, the "laboratory frame" refers to the unique frame in which the flow is stationary since boundary conditions and, in some cases, slow variations of the mean flow have broken the Galilean invariance.

The criterion for nonlinear absolute instability may be simplified further by considering the minimum speed of all the fronts separating the basic state from a saturated solution downstream. When this speed is negative (resp. positive) the instability is nonlinearly absolute (resp. nonlinearly convective). The selection problem for front velocity is well understood from the studies of Kolmogorov, Petrovsky and Piskunov [20], Dee and Langer [21], Dee [22], Ben-Jacob et al. [23], van Saarloos [24-26]), Powell, Newell and Jones [27] and of van Saarloos and Hohenberg [28] etc. on amplitude equations. By studying the GinzburgLandau equation, Dee and Langer [21] observed, to their surprise, that the front moves at the speed of the edge of the linear wave packet. This case is presently referred to as a pulled front since the linear region upstream of the front selects the velocity and the oscillation frequency of the entire nonlinear solution. Van Saarloos and Hohenberg [28], in particular, established that this was not always the case and that, in specific circumstances, the front moves faster upstream than the pulled front. This case is referred to as a pushed front and Chomaz and Couairon [29] showed that the saturated wave downstream of the front sets the frequency and the propagation speed of the entire solution. The region upstream of the front is then made of an evanescent wave driven by the nonlinear front. This linear wave is a so-called $k^{-}$-wave selected by causality since in the frame of the front the flow is convective (Chomaz and Couairon [29]). The remarkable feature is that, when the front is pulled, the linear absolute instability and the nonlinear absolute instability coincide. But for each particular flow, one should first determine

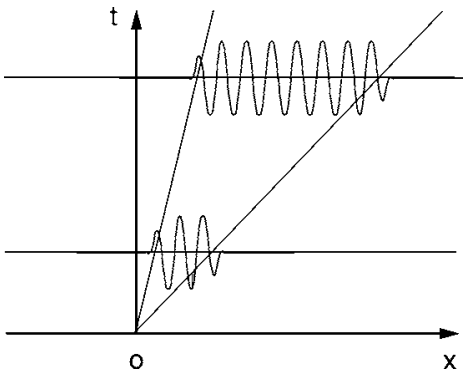

(a)

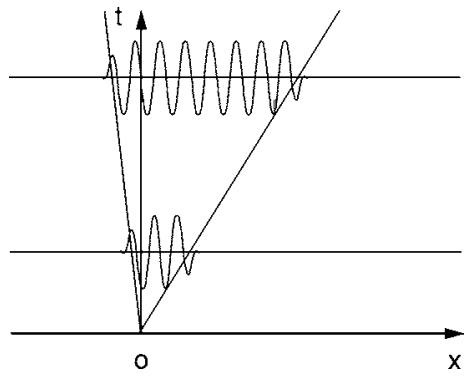

(b)

Fig. 1. Diagrams in the $(x, t)$ plane, displaying the dynamics of a saturated wave packet in an unstable flow, (a) nonlinear convective instability, the velocity $v_{f}$ of the trailing front separating the saturated wave from the basic state is positive, (b) nonlinear absolute instability, the front velocity $v_{f}$ is negative. 


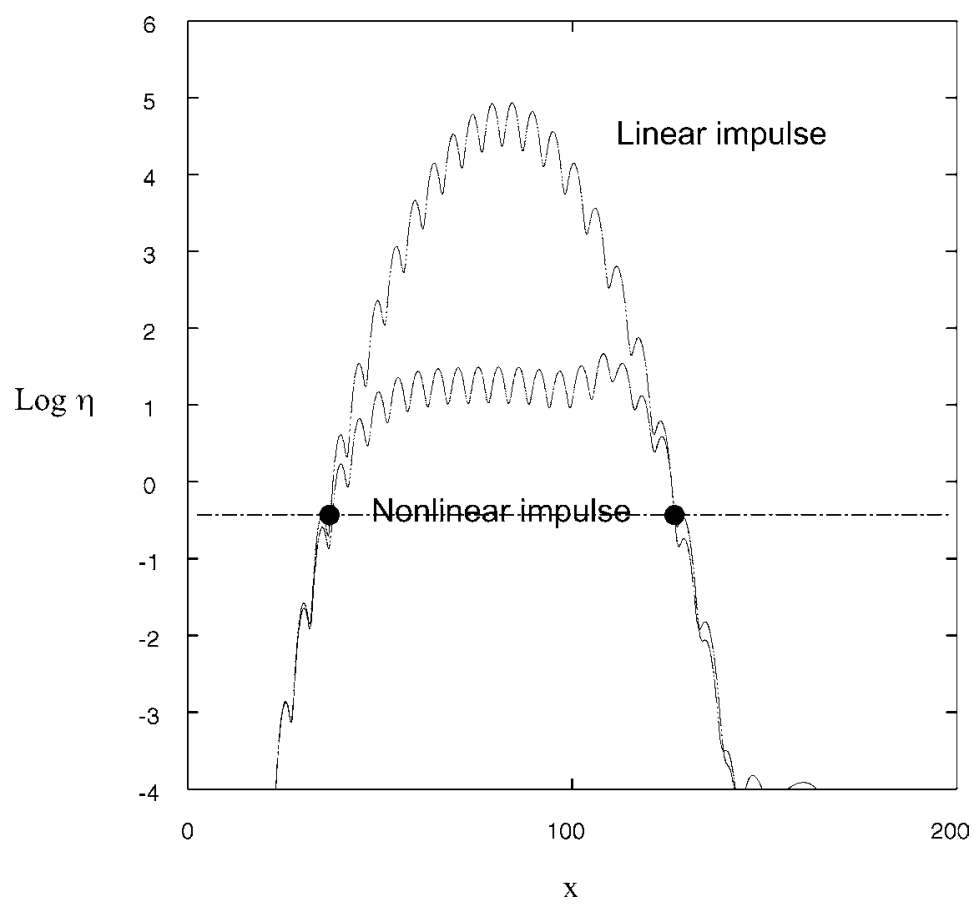

Fig. 2. Comparison of the linear and nonlinear impulse responses for the wake at $R e=20$. The enstrophy at time $t=100$ is plotted as a function of $x$. Only a small portion of the computational domain is presented.

if a pushed front exists or if the fastest front is a pulled front. For wakes, this study has been performed by Delbende and Chomaz [30]. Fig. 2 presents the local enstrophy associated with the linear and nonlinear wave packets obtained from the same initial impulsive perturbation in a Bickley wake, similar to the one studied by Delbende and Chomaz [30]. The basic wake profile was introduced by Monkewitz and Nguyen [31]:

$$
U_{B}(y)=2+U_{0}-\frac{2}{1+\sinh ^{2 N}\left|y \sinh ^{-1}(1)\right|},
$$

where $U_{0}$ is the centre line velocity, $U_{\infty}=2+U_{0}$ is the velocity at $y=\infty$. The velocity ratio used by Monkewitz and Nguyen [31] is then $\Lambda=\left(U_{0}-U_{\infty}\right) /\left(U_{0}+U_{\infty}\right)=-\left(1+U_{0}\right)^{-1}$ and $N$, the parameter that controls the steepness of the shear layer is fixed to 2 in the remainder of the study. A body force is added to the Navier Stokes equation to annihilate the basic flow diffusion (see appendix for details). With this artificial force, the basic flow becomes an exact solution of the equation and the perturbations are then given by an evolution operator homogeneous in time. Such a trick is systematically used to analyse the linear instability of viscous shear flow (cf. Drazin and Reid [32]).

The nonlinear impulse is limited by two sharp fronts that coincide with the edges of the linear wave packet. The front, separating the von Karman saturated street from the unperturbed stationary wake, is, therefore, a pulled front. At higher Reynolds number, pairing instabilities develop inside the nonlinear wave packet (Fig. 3) but they do not seem to affect the propagation speed, since both the upstream and the downstream fronts coincide with the edges of the linear impulse response.

Fig. 4 shows similar results for the parallel mixing layer with a hyperbolic tangent velocity profile:

$$
U_{B}(y)=U_{0}-\tanh y,
$$

where $U_{0}$ is the centre line velocity, $U_{\infty}=1+U_{0}$ is the velocity at $y=\infty$. In order to compare the results with those of Huerre and Monkewitz [33], we will use the velocity ratio $\Lambda=-1 / U_{0}$. As for the wake, a body force is added to the NavierStokes equation to annihilate the basic flow diffusion (see appendix for details). The linear impulse response exhibits the usual Gaussian envelope. The associated perturbation vorticity field is plotted in Fig. 5(a). The nonlinear impulse response is also plotted in Fig. 4. The edges of the nonlinear wave packet that may be defined as the location where the enstrophy is dropping to zero, are superimposed on their linear counterpart which means that the front is a pulled front. But the wave packet develops tails on both sides that correspond to the potential perturbations due to the pairing events that are visible on Fig. 5(b). These potential tails were already reported by Delbende and Chomaz [30]. Their impact on the dynamics is believed to be weak since the nonlinear wave packets are damped in the tail region, the damping occurring at a lower rate than for their linear counterpart. 
(a)

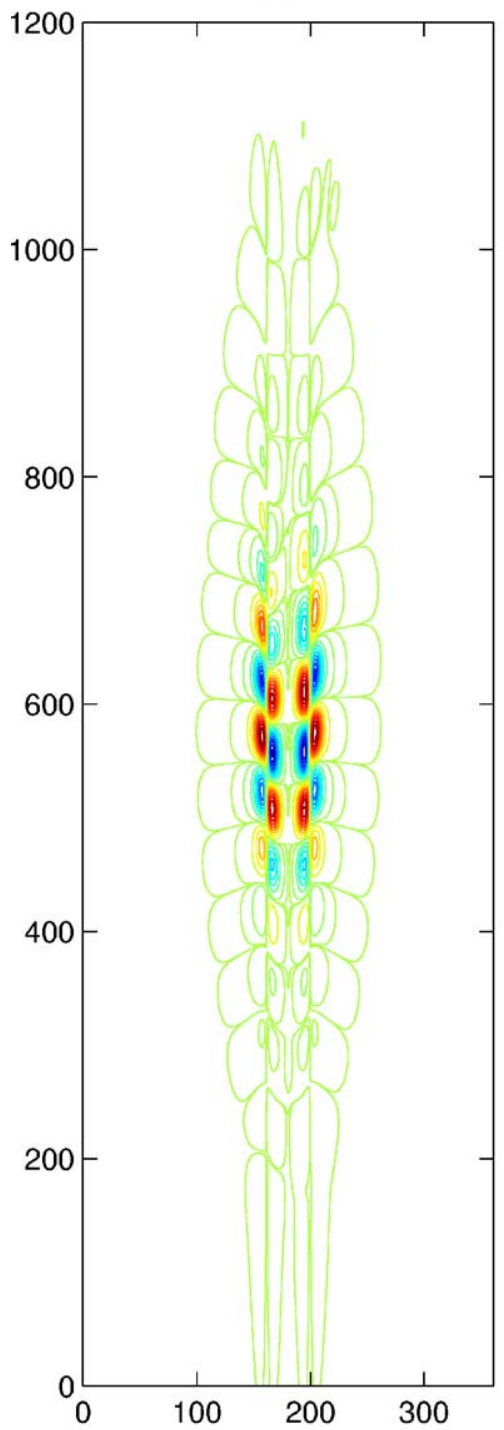

(b)

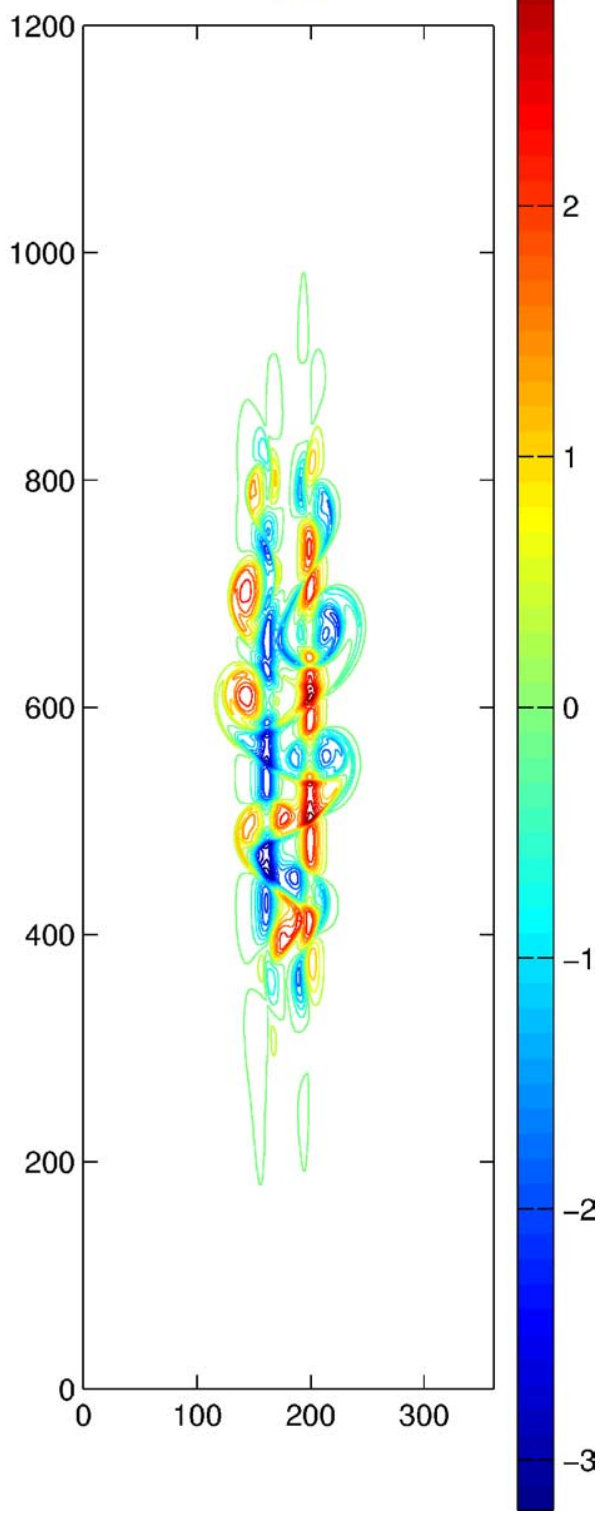

3

Fig. 3. Comparison of the linear (a) and nonlinear (b) impulse responses for the wake at $R e=4000$. The scales in $x$ and $y$ are measured in number of grid points and should be divided by 10 to correspond to the physical scales. The perturbation vorticity fields at time $t=25$ are plotted and only a small portion of the computational domain is presented. In the linear case (a), the outermost green iso-contour approximately indicates the edges of the impulse response, the amplitude of which is five orders of magnitude smaller than the inner part of the wave packet in red and blue. In the nonlinear case (b), the amplitude of the impulse response has saturated on the edges and is of order unity everywhere inside the two limiting fronts. Since the Reynolds number is large, pairings occur in the nonlinear case (b). Please note that the amplitude of the linear response has been divided by $10^{5}$ to be plotted with the same scale as the nonlinear response.

When comparing Figs. 5(a) and 5(b), the reader should be aware that the linear wave packet was normalized by $10^{-5}$ in order to use the same colorbar for both the linear and nonlinear response. The linear wave packet appears smaller because the most visible part is the inner one, whose amplitude is extremely large. To compensate for this effect, extra iso-contours (in green) corresponding to the amplitude close to the edge of the wave packet (i.e., $10^{-5}$ smaller than the largest amplitude) were drawn and they seem to correspond reasonably well with the boundary of the nonlinear wave packet. 


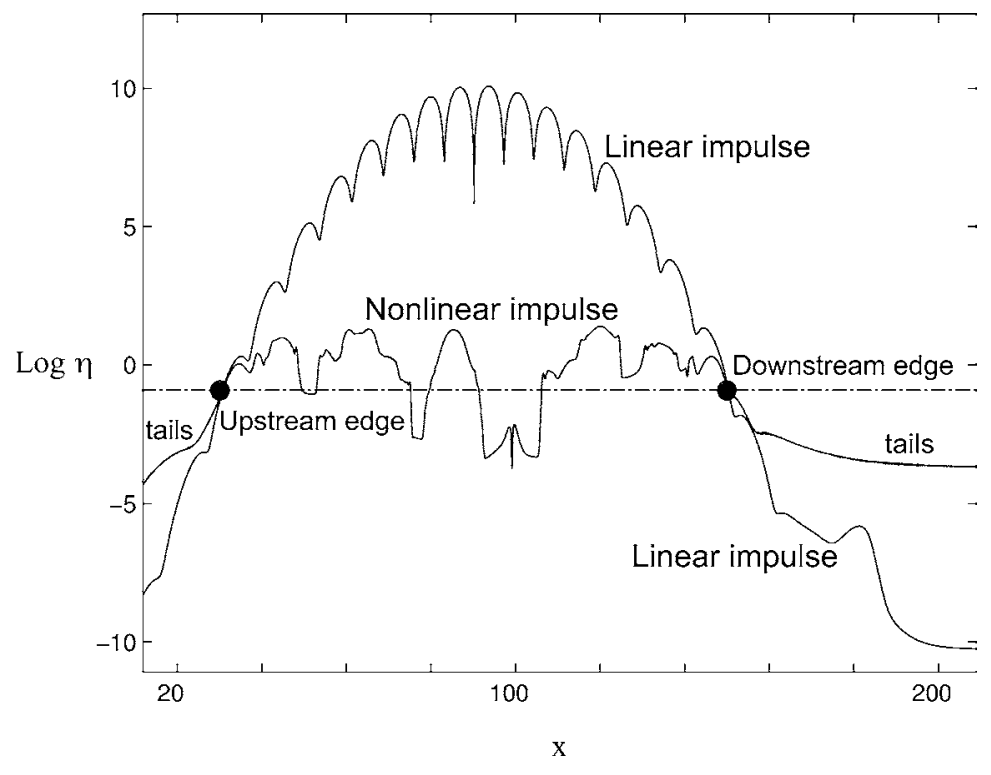

Fig. 4. Same as Fig. 2 but for the mixing layer. Comparison of the linear and nonlinear impulse response for the mixing layer at $R e=2000$. The enstrophy $\eta$ at time $t=97$ is plotted as a function of $x$. The fronts are located where $\eta$ drops to zero (materialized here by the dash-dotted line at $\log \eta=0.8$ and they coincide with the edges of the linear wave packet.

\section{Nonlinear Global Mode in parallel flow}

The previously shown impulse responses describe the dynamics in an infinite domain. In order to approximate numerically an infinite domain, these responses were computed in extremely long boxes (409.6 times the shear layer thickness or the wake width) and the computations were stopped before the domain became totally invaded by the perturbation. Experimentally a mixing layer (resp. a wake) is formed at the end of a splitter plate (resp. a cylinder) that imposes inlet boundary conditions on the flow and breaks the Galilean invariance. When the Reynolds number is large enough the viscous diffusion of the basic flow could reasonably be neglected. In that case, the basic flow is parallel at leading order with a streamwise velocity profile that does not evolve downstream. Still the fact that the inlet condition singles out a particular Galilean frame considerably modifies the dynamics. As proposed by Chomaz [19], if the basic flow is nonlinearly convective, any initial perturbations should eventually vanish since the response it triggers should be limited upstream by a front that moves away from the inlet. On the contrary, if the instability is nonlinearly absolute, a self-sustained oscillation (a nonlinear Global Mode) should occur, the speed of the front limiting the nonlinear response being then negative in the laboratory frame. This result was confirmed by analysing solutions of several amplitude equations in a semi-infinite domain with the condition of vanishing amplitude at the inlet (Couairon and Chomaz [34-37]). When, for a particular amplitude equation, a pushed front is selected, a self-sustained oscillation occurs before the flow becomes linearly absolutely unstable, i.e. while it is linearly stable or convectively unstable. This is because a pushed front moves faster upstream than the edge of the linear impulse and therefore the velocity of the front becomes negative while the velocity of the edges of the linear wave packet is still positive. In such a case the selected frequency is that of the nonlinear front. It is nonlinearly selected and cannot be inferred from characteristics of the linear dispersion relation.

On the contrary, when the front is pulled, its speed equals that of the edges of the linear wave packet and linear and nonlinear absolute instability thresholds coincide. In that case, a nonlinear Global Mode bifurcates when the instability is linearly absolute. At the threshold the selected frequency is then the absolute frequency $\omega_{A}$, the frequency of the linear wave with a zero group velocity (see Huerre and Monkewitz [6]).

No matter if the front is pulled or pushed, the structure of the nonlinear Global Mode is similar. As the control parameter is increased above this critical value at which the instability becomes nonlinearly absolute, the Global Mode associated with the self-sustained oscillation saturates closer to the inlet. The Mode is described at leading order by a front which, without any boundary, would have moved upstream and which is presently blocked by the inlet condition.

Fig. 6(a) presents an example of a Global Mode obtained just above the absolute instability threshold by a numerical integration of the Ginzburg-Landau amplitude equation with complex coefficients (see Couairon and Chomaz [37] for details). The interpretation in terms of a front separating a saturated wave from the basic state is straightforward. Dee and Langer [21] showed that fronts in the complex Ginzburg-Landau equation were pulled and Couairon and Chomaz [37] have confirmed that, in that case, the global bifurcation occurred as soon as the basic state became absolutely unstable (Fig. 6(b)). At threshold, the 
(a)

(b)
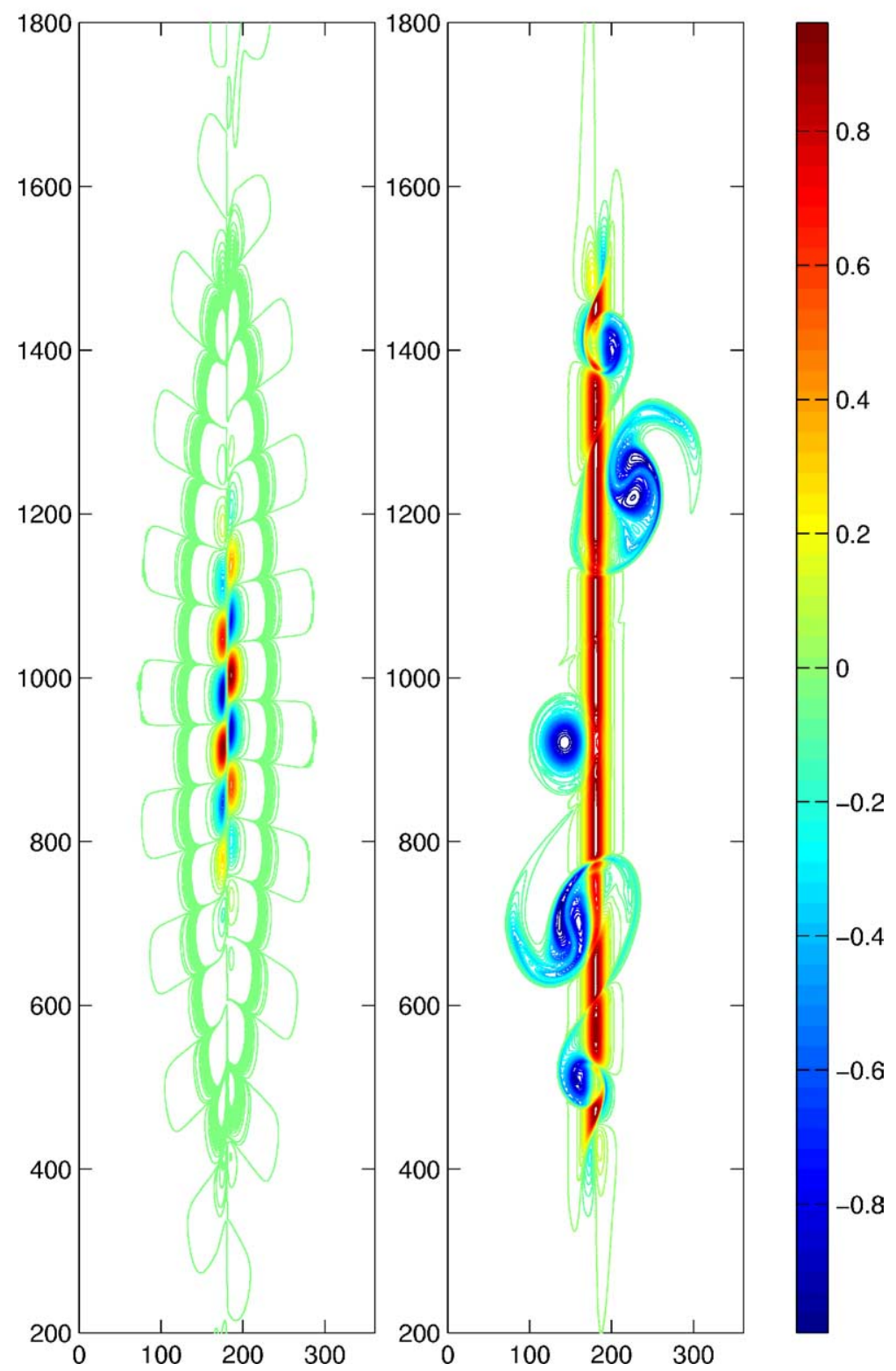

Fig. 5. Same as Fig. 3 but for the mixing layer. Comparison of the linear (a) and nonlinear (b) impulse responses for the mixing layer at $R e=2000$ and time $t=90$. For this very late time many pairings have occurred and the wavelength has increased even at the edges of the nonlinear impulse response. The scales in $x$ and $y$ are measured in number of grid points and should be divided by 10 to correspond to the physical scales.

global frequency $\omega_{G}$ of the self-sustained oscillation is equal to the absolute frequency $\omega_{A}$, the frequency of the wave with zero group velocity.

Tobias, Proctor and Knobloch [38,39] obtained similar results when studying numerically the super-critical GinzburgLandau equation. Fineberg and Steinberg [40], and Müller et al. [41,42] observed the same dynamics in Rayleigh-Bénard convection with through-flow and Ahlers and Cannel [43] and Büchel et al. [44] in Taylor-Couette experiments with throughflow. In the two latter cases, the basic flow is parallel since viscous diffusion of the through-flow is balanced by a pressure gradient. Depending on the inlet condition, the basic flow may vary in a short domain close to the inlet before the balance is locally realized everywhere, but the previously quoted authors showed that this effect, when it exists, is negligible. 


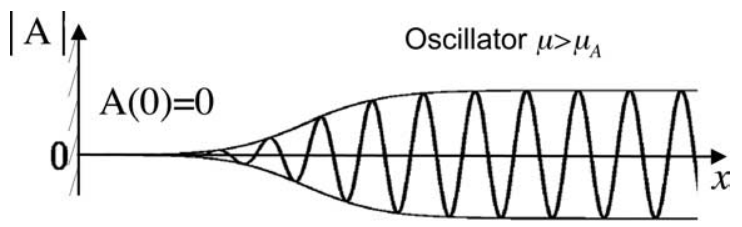

(a)

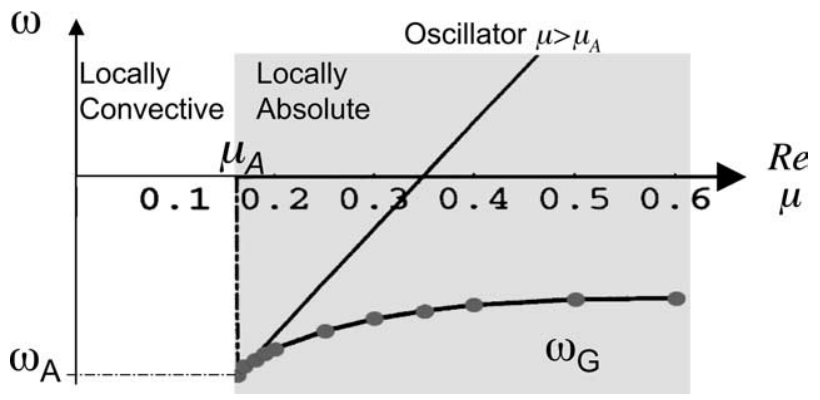

(b)

Fig. 6. (a) Global Mode obtained above the absolute instability threshold but very close to it, by numerical integration of the Ginzburg-Landau amplitude equation with complex coefficients (see Couairon and Chomaz [37] for details). (b) The bifurcation diagram showing the frequency of the Global Mode as a function of the control parameter $\mu$ (dark grey bullets). In the grey domain the instability is absolute. Oscillations are self-sustained only when the instability is absolute.

For wakes, Triantafyllou and Karniadakis [45] demonstrated that the dynamics of the flow and its downstream evolution may be reproduced convincingly if, instead of computing the dynamics of the whole flow around the cylinder, the cylinder region was cut away and the flow computed in a semi-infinite domain. At the inlet (the upstream boundary of the computational domain), they imposed the axial velocity profile measured just behind the cylinder. But in their case, the basic flow was evolving in the streamwise direction under the action of the diffusion. They demonstrated that at the global instability threshold, the basic flow was absolutely unstable in a finite domain but the non-parallelism of the basic flow kept them from making quantitative comparisons with the theory. In particular, the critical Reynolds number where a region of absolute instability appears, preceded the threshold Reynolds number for the global instability by a value of order unity.

In the present study, we get rid of the extra complexity of the non-parallelism of the flow by adding a body force that exactly compensates for the diffusion of the basic flow. In that case, the behaviour of the flow is consistent with the scenario described above for the solutions of amplitude equations. Fig. 7(a) presents a nonlinear Global Mode resulting from the instability of a parallel wake in a semi-infinite domain with vanishing perturbations at the inlet. The Global Mode was computed as described in the appendix for $\Lambda=-0.909$. For this value of $\Lambda$, the basic flow is oriented downstream everywhere and no back flows are present. The Reynolds number is 400 , above the absolute instability threshold computed by Monkewitz [46]. The Global Mode is self-sustained, leading to the roll-up of the double shear layer into a stable vortex street that resembles a von Karman street. For such a large Reynolds number, the body force is small and its effect, barely noticeable, is that of a weak vorticity injection at the location of the unperturbed double shear. This nonlinear Global Mode may be analysed in terms of a front stopped by the inlet. After an initial growth, saturation occurs at a distance $\Delta_{x}$ from the inlet that decreases when the criticality is increased. This Global Mode exists only when the local instability is absolute. As an illustration, the bifurcation diagram for the wake with $\Lambda=-1$ corresponding to zero backflow, is plotted in Fig. 7(b). When the Reynolds number is larger than 45, the value of the absolute instability threshold determined by Monkewitz [46], a self-sustained oscillation appears. The maximum amplitude of this Global Mode, measured by its local enstrophy, saturates at the same finite value no matter how close we are to the threshold. Only the distance $\Delta_{x}$ at which saturation occurs varies with the departure from threshold. This distance is well fitted (Fig. 7(b)) by $\Delta_{x} \sim 60.2 \times\left(R e-R e_{A}\right)^{-1 / 2}$, the scaling law predicted by the theory (see Couairon and Chomaz [35] for details). The frequency of the self-sustained oscillation is then the absolute frequency (data not shown, see Chomaz [47], for details). In the previous examples, it should be noticed that the basic flow is always in the positive $x$-direction and that a backflow is therefore not necessary to obtain a self-sustained oscillation. Only the front separating the basic flow from the saturated state should move upstream, i.e., with a negative velocity.

The previous section on the dynamics of localized initial perturbations in an infinite mixing layer, demonstrates that the mixing layer sustains only pulled fronts and obeys, therefore, the Dee and Langer [21] selection principle. In that case, linear 


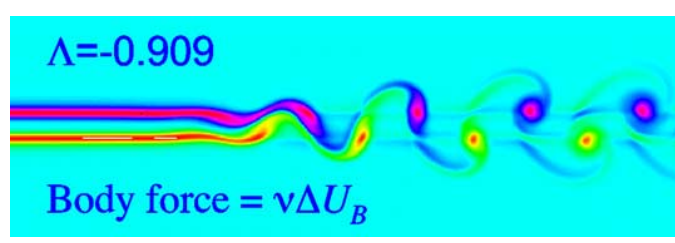

(a)

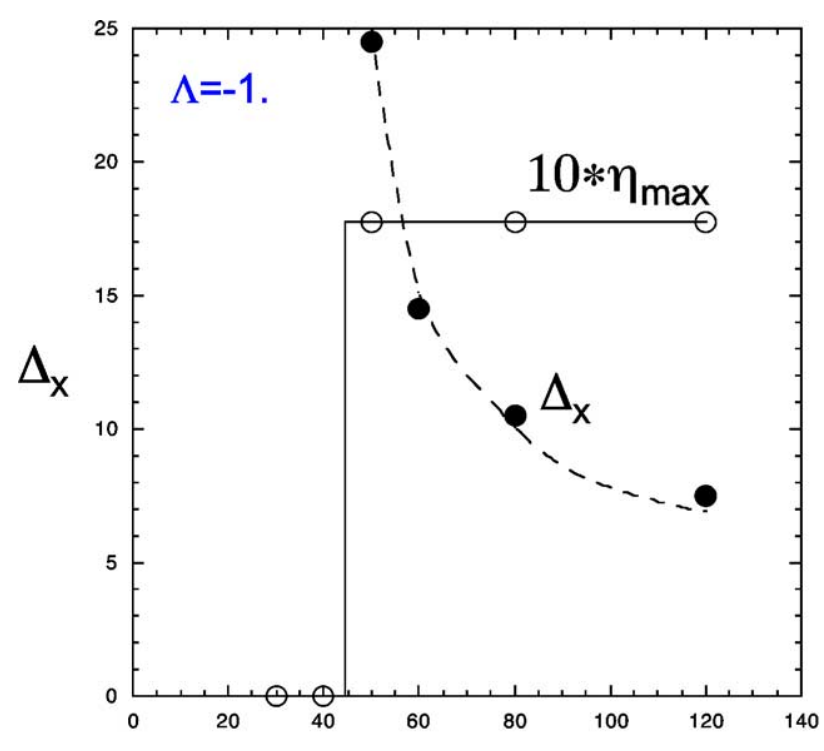

$\operatorname{Re}$

\section{$10 * \eta_{\max }$}

(b)

Fig. 7. (a) Global Mode of the parallel wake obtained for a Reynolds number $R e=400$ above the absolute instability threshold, for $\Lambda=-0.909$. The computational box is 16.8 large and 51.2 long, the wake width being unity. The sponge region, not shown here is 51.2 long, the total domain size being $102.4 \times 16.8$. (b) Bifurcation diagram for the wake, showing, for $\Lambda=-1$ (zero backflow) the saturation local enstrophy $\eta_{\max }$. Following Monkewitz [46] the instability becomes absolute for $R e$ larger than $R e_{A} \sim 45$. We observe that, indeed, when the instability becomes absolute the amplitude of the Global Mode measured by $\eta_{\max }$ suddenly increases from zero to a constant finite value as predicted by Couairon and Chomaz [35]. The distance $\Delta_{x}$ at which saturation occurs, defined by the location where $\eta(x)=1$, is also plotted and it is well fitted by $\Delta_{x} \sim 60.2 \times\left(R e-R e_{A}\right)^{-1 / 2}$ as predicted by the theory.

and nonlinear absolute instability criteria coincide and the fully nonlinear global bifurcation should occur when the instability is locally linearly absolute. This is indeed the case and the Global Mode that appears is illustrated in Fig. 8 . The self-sustained state in Fig. 8 is obtained for $R e=400$ and $\Lambda=-1.42$, which is above the absolute instability threshold, $\Lambda_{A}=-1.315$ determined by Huerre and Monkewitz [33] for $R e=\infty$. When $\Lambda=-1.25$, oscillations are not self-sustained (data not shown) in agreement with the fact that the flow is then convectively unstable.

The Global Mode for the mixing layer does not seem to be stable: according to Fig. 8, pairings occur even for parameter values close to the absolute instability threshold and although pairing is slightly inhibited by the lateral confinement. For the same Reynolds number $R e=400$, the wake behaves differently and a stable wake is obtained with no pairing occurring as shown in Fig. 7. This different behaviour corresponds to distinct characteristics of the secondary instability and it will be commented on in Section 5.

\section{Nonlinear Global Mode in non-parallel flow}

For wakes or mixing layers, the parallel basic flow approximation is valid only if the viscosity is nil or compensated by an artificial body force. But in numerical or laboratory experiments, viscosity is finite and the basic flow imposed at the inlet evolves downstream. When the non-parallelism of the real flow is weak enough, i.e. when the scale on which the basic flow varies is large enough compared to the typical scales of the instability (the wavelength, and/or the inverse of the spatial growth rate), the present results may be extended in a straightforward manner. In that case, a nonlinear self-sustained oscillation occurs 


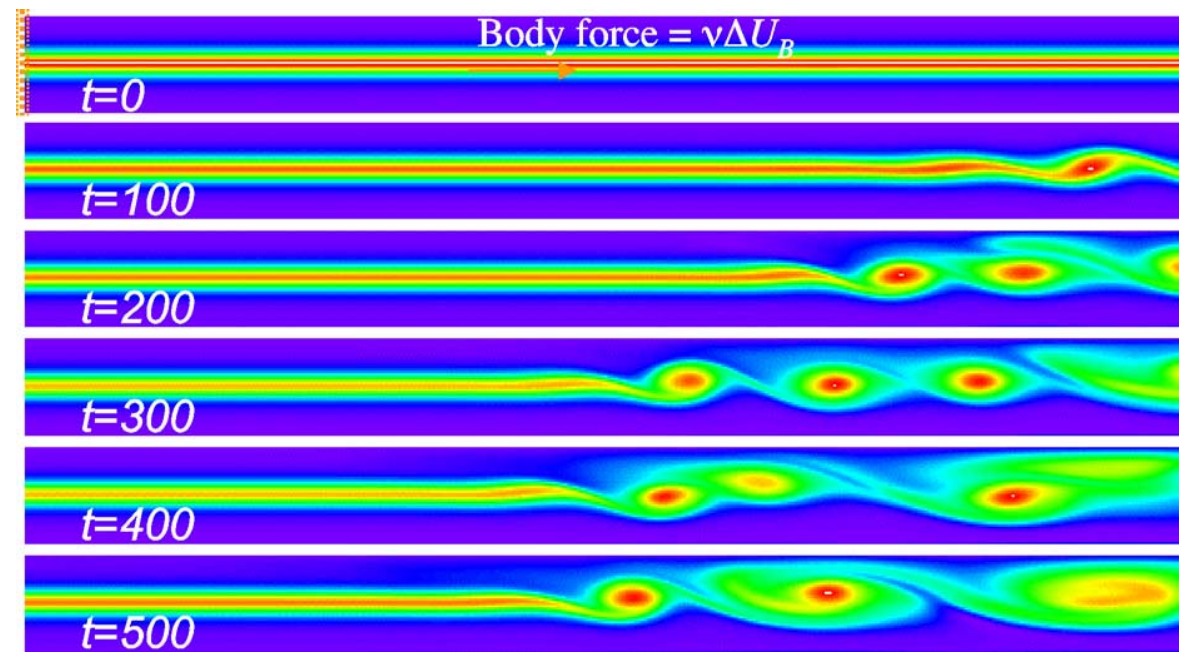

Fig. 8. Time series showing the establishment of a Global Mode for the parallel mixing layer obtained at Reynolds number 400 for a velocity ratio $\Lambda=-1.42$, above the absolute instability threshold $\Lambda=-1.315$. The computation box is 8.4 large and 102.4 long, the mixing layer width being unity. The sponge region, not shown here is 102.4 long, the total domain size being $204.8 \times 8.4$.

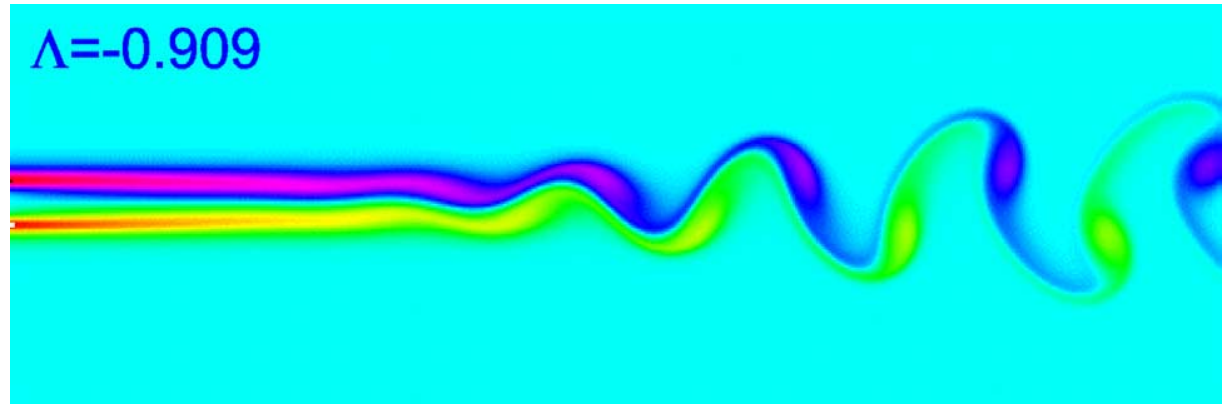

Fig. 9. Global Mode of the nonparallel wake obtained for a Reynolds number $R e=800$ for $\Lambda=-0.909$, i.e., when the inlet flow is everywhere positive. This Reynold number is well above the absolute instability threshold $R e_{A} \sim 125$ for this particular value width being unity. The sponge region, not shown here is 51.2 long, the total domain size being $102.4 \times 16.8$.

as soon as a finite domain of absolute instability is present and it is made up of a front located at the most upstream border of the absolutely unstable domain. The oscillation frequency of the Global Mode is then the absolute frequency at the front location. This border may be either a physical boundary of the flow, as discussed by Chomaz [19], Couairon and Chomaz [34-37,5,48], Worledge et al. [49], Tobias, Proctor and Knobloch [38,39], Chomaz and Couairon [50], and Soward [51] or a location where the flow goes from absolutely unstable to convectively unstable as explained in Pier et al. [52], Pier, Huerre and Chomaz [53], Harris, Bassom and Soward [54] and in Pier and Huerre [55]. Fig. 9 shows an example of a nonlinear Global Mode in a weakly non-parallel wake obtained by turning on the diffusion of the basic flow in the model. For the wake with $\Lambda=-0.909$, the instability threshold is postponed to larger values of the Reynolds number but the frequency selection is unchanged. For the mixing layer, when non-parallel effects are added, the global oscillation is replaced by a steady flow (not shown), in which the mixing layer is sucked in by the backflow as described by Hammond and Redekopp [8].

\section{Stability of nonlinear Global Mode in parallel flow}

As discussed in Section 3, when the flow is parallel in a semi-infinite domain, a nonlinear Global Mode is made of a steady front followed by a saturated nonlinear state. The closer to the threshold, the longer it takes to reach saturation, but no matter how close to the threshold, saturation still occurs. This fully nonlinear instability scenario is only the first step towards transition and it may be repeated for the secondary bifurcation (Couairon and Chomaz [4], Chomaz, Couairon and Julien [56]), since the finite amplitude wave that follows the front may be itself absolutely or convectively unstable. The region of the parameter 
space where that finite amplitude wave is absolutely unstable will be designated by the acronym ASI for absolute secondary instability and CSI (convective secondary instability) will designate the convectively unstable domain. By studying the solution of amplitude equations, different routes have been identified, depending on which of the primary or the secondary instabilities of the selected saturated wave first becomes absolutely unstable.

When the saturated state generated by the primary absolute instability (for example the saturated vortex street) is convectively unstable (CSI) when the primary instability becomes absolute, the bifurcation scenario is relatively classical. In particular, a single frequency appears at threshold and, if the control parameter is further increased, so that the secondary instability becomes, itself, absolutely unstable, a second frequency appears. This case is illustrated by the dynamics of the complex GinzburgLandau equation (Couairon and Chomaz [4]) in Fig. 10, where the modulus of the Global Mode is plotted versus $x$. Below the threshold for secondary absolute instability (Fig. 10(a)), the initial transient amplitude oscillations are washed downstream and the envelope will eventually be steady and saturate at finite value. Above the threshold, the amplitude oscillation never stabilizes (Fig. 10(b)) and the mode oscillates at two frequencies.

When the saturated wave is absolutely unstable with respect to secondary perturbations at the Global Mode threshold, perturbations in the lee of the primary front never settle down and the first bifurcation gives rise directly to a two-frequency mode (Fig. 11). In the complex Ginzburg-Landau equation, either the first or the second scenario occurs by simply varying the complex diffusion and nonlinear coefficients (see [4], for details). When the secondary instability is strongly absolute, it is even possible to find an erratic behaviour at the first instability threshold.

The nature of the secondary instability was computed for the Stuart nonlinear solution [57] that represents a single row of finite size vortices mimicking a mixing layer after roll-up, by Brancher [5] and Brancher and Chomaz [3] using direct numerical simulation of 2D or 3D impulse responses as described in the first section. The family of 2D basic flows solution of the Euler equation proposed by Stuart [57] is defined by the stream function:

$$
\Psi_{b}(x, y)=\frac{1}{2} \ln [\cosh (2 y)-\rho \cos (2 x)] .
$$

This solution describes a $\pi$-periodic array of two-dimensional vortices separating two counter flows $u_{x}(y= \pm \infty)= \pm 1$. This family of solutions is characterized by the non-dimensional parameter $\rho \in[0,1]$ which is a measure of the vortex concentration. The solution for $\rho=0$ corresponds to the parallel tanh velocity profile (infinitely spread vortices) and the solution $\rho=1$ consists of a periodic street of point vortices (infinitely concentrated vortices). The temporal stability of the Stuart vortex street has been studied by Pierrehumbert and Widnall [58] and more recently Potylittsin and Peltier [59]. Brancher [5] and Brancher and Chomaz [3] have performed the spatio-temporal stability analysis and found, both for the two- and threedimensional instabilities, that the more concentrated the vortices, the less the backflow needed to trigger the absolute instability (Figs. 12 and 13). In particular, for all the concentration parameters tested, the backflow needed to have a secondary absolute instability (2D or 3D) is smaller than the one needed to have a primary absolute instability $(\Lambda=-1.315)$. For the mixing layer, the secondary pairing and the translative instabilities therefore become absolute sooner than the primary Kelvin Helmholtz instability.

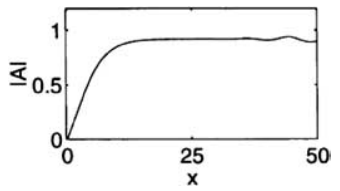

(a)

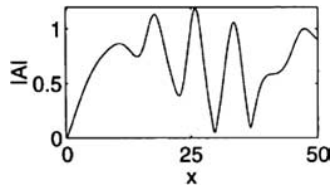

(b)

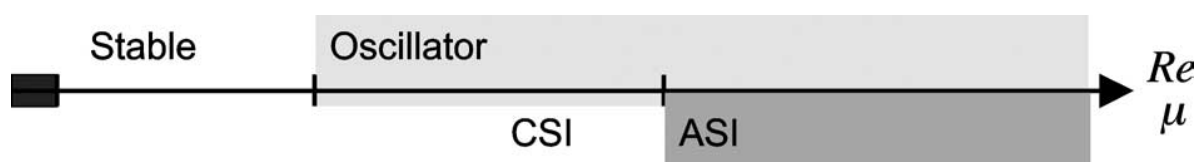

(c)

Fig. 10. (a) Global Mode envelope obtained above the primary absolute instability threshold when the secondary instability is convectively unstable (CSI), by numerical integration of the Ginzburg-Landau amplitude equation with complex coefficients (see [4] for details). The small perturbation on the right of the domain is a leftover of the starting transient which was initially amplified but was moving to the right and which will eventually vanish. (b) Same mode when the control parameter $\mu$ is increased above the secondary absolute nstability threshold (ASI), oscillations of the amplitude are permanent. (c) Schematic bifurcation diagram summarising the first scenario, when the control parameter ( $\mu$ for the model or, for example, $R e$ for the wake) is increased the Global mode (Oscillator) appears when the primary instability becomes absolutely unstable (light grey domain). The secondary instability of the saturated wave that constitutes the global mode far from the inlet region becomes itself absolutely unstable in the dark grey region (ASI) and is convectively unstable before (CSI). Case (a) pertains to the CSI domain and case (b) to the ASI domains. 


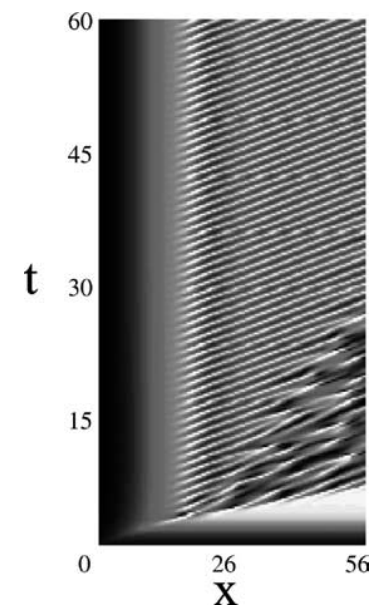

(a)

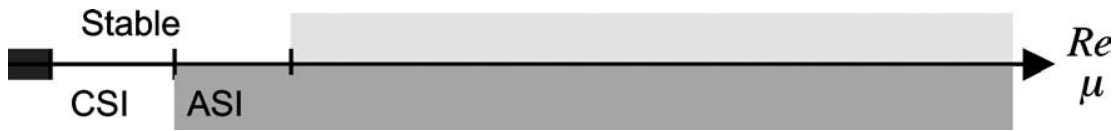

(b)

Fig. 11. (a) Spatio-temporal $(x-t)$ diagram of the Global Mode at threshold showing its amplitude as a function of inlet distance $x$ and time $t$. Oscillations of the amplitude indicated by the black and white strips, are permanent as soon as the Global Mode exists. This is because the absolute primary instability threshold that leads to the occurrence of the selfsustain structure, is exceeded when the secondary instability is already absolute (ASI). (c) same as Fig. 10(c), schematic bifurcation diagram summarising this second scenario, when the control parameter ( $\mu$ or $R e$ ) is increased the Global mode appears (light grey domain) whereas the saturated wave is already absolutely unstable (dark grey region, ASI). The global mode that appears is more complex than a simple oscillator (a two frequency state on the spatio-temporal diagram (a)).

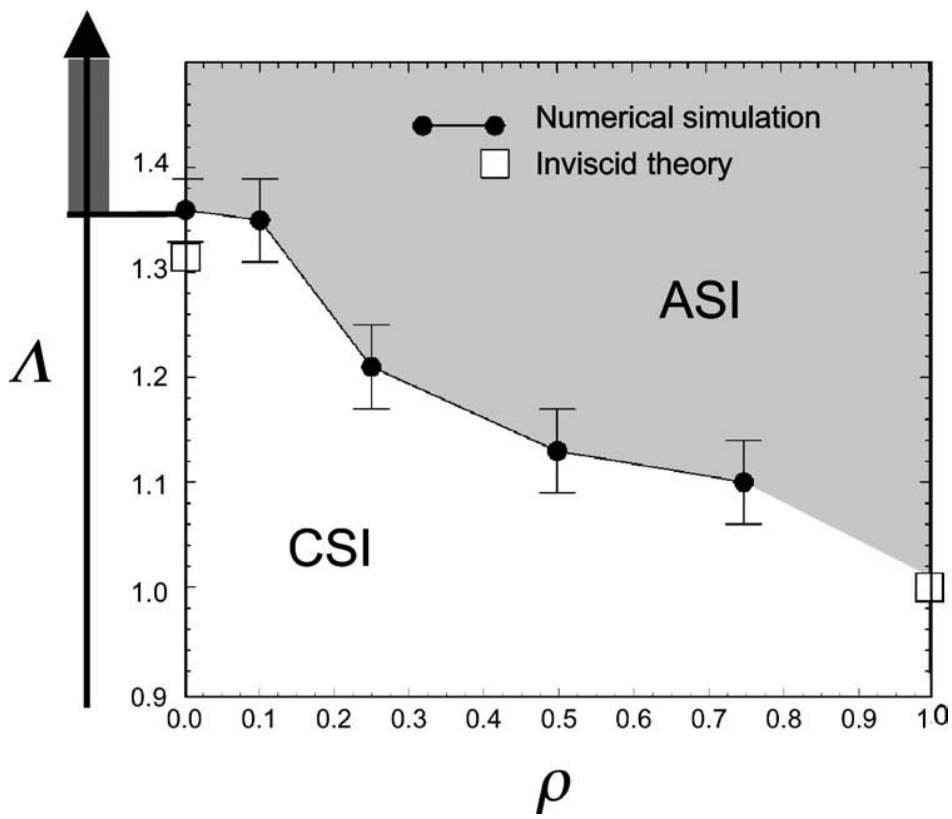

Fig. 12. Domains of absolute (A) and convective (C) 2D pairing instability in the $(\Lambda, \rho)$ plane. The pairing instability becomes absolute for a smaller backflow than the primary instability (see [3] for details). 


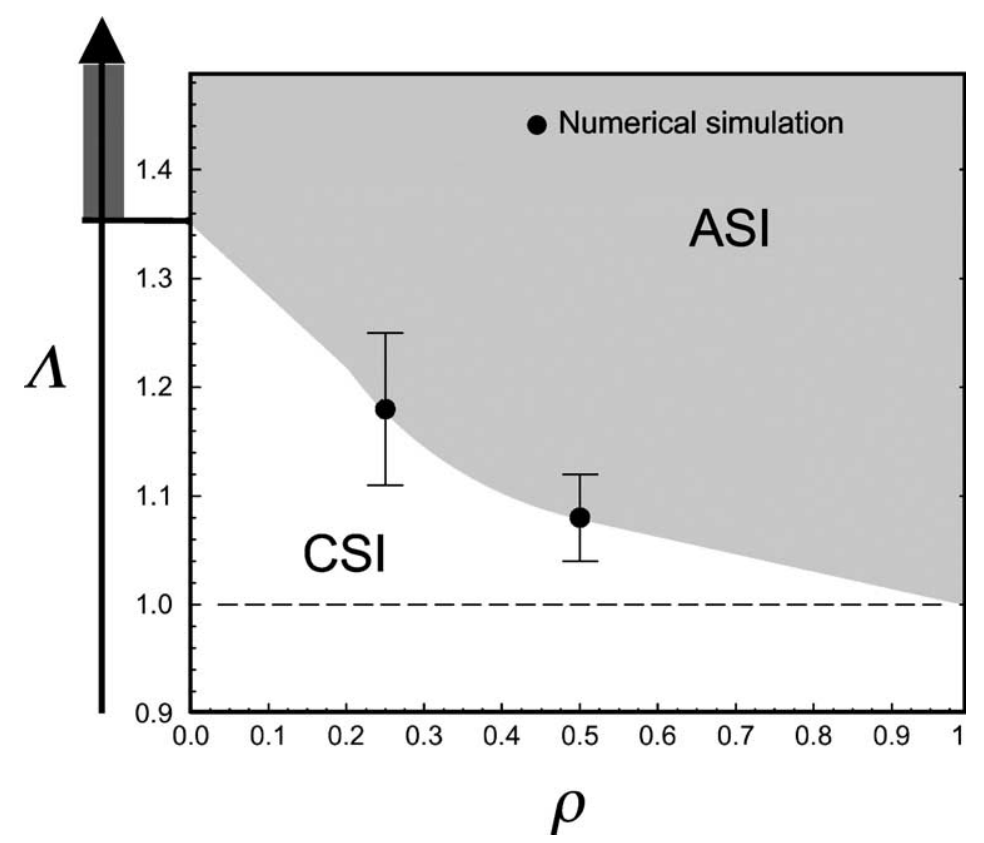

Fig. 13. Domains of absolute (A) and convective (C) 3D translative instability in the $(\Lambda, \rho)$ plane. The spanwise wave number is fixed to $k=2$. The translative instability becomes absolute for a smaller backflow than the primary instability (see [5] for details).

These results on the pairing instability explain why the 2D Global Mode in the parallel mixing layer presented in Fig. 8 is irregular at its apparition threshold with pairings occurring randomly. We may now propose an alternative interpretation based on the secondary absolute instability scenario for the subharmonic resonance observed in forced jets or backward facing step flows, which is classically attributed to an acoustic feedback (Broze and Hussain [60], Narayanan and Hussain [61], Kaiktsis, Karniadakis and Orszag [62]). It also predicts that three-dimensional instability may become resonant and therefore extremely intense when the primary instability is forced or is self-sustained. This might explain the occurrence of intense side jets in round jet experiments (Monkewitz et al. [12]).

Eventually these new scenarii may explain brutal transition to turbulence as observed in the rotating disk and discussed by Koch [63], Brandt et al. [64] and Pier [65].

\section{Conclusion}

In conclusion, the fully nonlinear extension of the concepts of absolute and convective instability based on front solutions (Chomaz [19]) is used to analyse the dynamics of wave envelop models and of actual flows such as wakes and mixing layers which are kept parallel by compensating the viscous diffusion of the base flow by the addition of the appropriate body force. In all the cases studied, the front speed is first shown to be equal to the linear wave packet edge (pulled fronts). Then, when inlet conditions are introduced a fully nonlinear global mode is shown to appear when the flow is absolutely unstable in the reference frame singled out by the inlet condition. The global mode oscillates then at the absolute frequency and is made of a front stop at some distance from the inlet. Both for the wake and the mixing layer, the distance at which saturation occurs varies as the square of the inverse of the criticality (the departure from threshold). Since a global mode in a semi-infinite parallel flow reaches finite amplitude saturation at threshold it may itself be unstable. This will occurs when the secondary instability of the uniform saturated wave that follows the front is absolute. But the secondary instability may be absolute either before or after the primary. In the first case a stable global mode will appear first and then, if the control parameter is further increased, it may be destabilized if the secondary instability becomes absolute. In the second case the global mode is already unstable at threshold and an oscillatory or even a chaotic state appears after a single bifurcation. This second scenario involving secondary absolute instability may explain a one step transition to disorder or turbulence as observed for example in rotating disk or mixing layers experiments.

It is worth mentioning that other scenarii should exist that involve a pulled front and for which linear theory should not give a correct prediction. Since the numerical technique introduced by Delbende, Chomaz and Huerre [66] and Delbende and Chomaz [30], allows the direct determination of the linear and nonlinear absolute or convective nature of instabilities, 
investigations of many different flows are now in hand. Hopefully, some of them will exhibit a nonlinear front velocity selection (pulled front) for which strong departure from linear prediction has already been demonstrated on model equations. In fluids, a nonlinear absolute instability was observed recently for the shear instability in an Hele-Shaw cell (Gondret et al. [67]), the other observations being presently limited to chemical reactions (Hanna, Saul and Showalter [68]).

\section{Appendix. The numerical procedure}

The numerical procedure is similar to that used in Delbende and Chomaz [30]. It integrates the perturbed Navier-Stokes equations:

$$
\left.\begin{array}{l}
\frac{\partial \omega}{\partial t}=J(\psi, \omega)-U_{B} \frac{\partial \omega}{\partial x}+\frac{\partial \phi}{\partial x} \frac{\partial \Omega_{B}}{\partial y}+v \Delta \omega, \\
\omega=-\Delta \psi, \\
J(\psi, \omega)=\frac{\partial \phi}{\partial x} \frac{\partial \omega}{\partial y}-\frac{\partial \omega}{\partial x} \frac{\partial \phi}{\partial y},
\end{array}\right\}
$$

where $\Omega_{B}=-\mathrm{d} U_{B} / \mathrm{d} y$ is the basic flow vorticity, $u=u e_{x}+v e_{y}$ the perturbation velocity, $\omega e_{z}=\nabla \times u$ the perturbation vorticity, and $\phi$ the stream function such that $u=\frac{\partial \phi}{\partial y}$ and $v=-\frac{\partial \phi}{\partial x}$. The viscous dissipation of the basic flow $U_{B}$ is, in the above formula, compensated exactly by a body force $-v \Delta U_{B}$. The parallel basic flow is then an exact solution. For non-parallel flow simulation the body force is suppressed and the viscous dissipation term $v \Delta U_{B}$ added to Eq. (4).

The equations are integrated in a very long box of size $2 L \times H$, periodic in the $x$-direction and with free slip boundary conditions in the $y$-direction.

To compute the dynamics of open flow, the fringe region technique used by Hogberg and Henningson [69], is implemented. Between $x_{\text {sponge }}$ and $2 L$ a linear damping term $-A(x) w(x, y, t)$ is added to the evolution equation (4). The damping function $A(x)$ is taken from Hogberg and Henningson [69]:

$$
\begin{aligned}
& x<x_{\text {sponge }}, \quad A(x)=0, \\
& x_{\text {sponge }}<x<x_{\text {sponge }}+\delta_{\mathrm{r}}, \quad A(x)=\frac{A}{1+\exp \left(\delta_{\mathrm{r}} /\left(x-x_{\text {sponge }}\right)+\delta_{\mathrm{r}} /\left(x_{\text {sponge }}+\delta_{\mathrm{r}}-x\right)\right)}, \\
& x_{\text {sponge }}+\delta_{\mathrm{r}}<x<2 L-\delta_{\mathrm{f}}, \quad A(x)=A, \\
& 2 L-\delta_{\mathrm{f}}<x<2 L, \quad A(x)=\frac{A}{1+\exp \left(\delta_{\mathrm{f}} /\left(x-L+\delta_{\mathrm{f}}\right)+\delta_{\mathrm{f}} /(L-x)\right)} .
\end{aligned}
$$

The damping intensity is determined by $A, \delta_{\mathrm{r}}$ is the length of the region where the damping grows from zero to $A$, and $\delta_{\mathrm{f}}$ is the length of the region where the damping relaxes back to zero. In practice, $x_{\text {sponge }}=L, \delta_{\mathrm{r}}$ equals $L / 2$ and $\delta_{\mathrm{f}}=0.5$. The value of $A$ is 10 in all simulations. This high value associated with a very fast drop of $A(x)$ on a few collocation points, allows an accurate simulation of the inlet condition where the amplitude of the perturbation should vanish.

As in Delbende and Chomaz [30], the dynamics of the flow is analysed using the enstrophy $\eta(x, t)$ computed at each streamwise location $x$ and time $t$ by:

$$
\eta(x, t)=\int_{-H / 2}^{-H / 2} \omega^{2}(x, y, t) \mathrm{d} y .
$$

\section{Acknowledgements}

The author warmly acknowledges P. Brancher, A. Couairon, I. Delbende, B. Pier and P. Huerre for fruitful discussions that motivated the present work.

\section{References}

[1] P. Strykowski, D. Niccum, The stability of countercurrent mixing layers in circular jets, J. Fluid Mech. 227 (1991) $309-343$.

[2] P.J. Strykowski, A. Krothapalli, S. Jendoubi, The effect of counterflow on the development of compressible shear layers, J. Fluid Mech. 308 (1996) 63-96.

[3] P. Brancher, J.-M. Chomaz, Absolute and convective secondary instabilities in spatially periodic shear flows, Phys. Rev. Lett. 78 (1997) 658-661. 
[4] A. Couairon, J.-M. Chomaz, Primary and secondary nonlinear global instability, Physica D 132 (1999) 428-456.

[5] P. Brancher, Étude numérique des instabilités secondaires de jets, Ph.D. thesis, École Polytechnique, France, 1996.

[6] P. Huerre, P.A. Monkewitz, Local and global instabilities in spatially developing flows, Annu. Rev. Fluid Mech. 22 (1990) $473-537$.

[7] C. Cossu, J.M. Chomaz, Global measures of local convective instability, Phys. Rev. Lett. 78 (1997) 4387-4390.

[8] D. Hammond, L. Redekopp, Global dynamics of symmetric and asymmetric wakes, J. Fluid Mech. 331 (1997) 231-260.

[9] B. Woodley, N. Peake, Global linear stability analysis of thin aerofoil wakes, J. Fluid Mech. 339 (1997) 239-260.

[10] T.-S. Leu, C.-M. Ho, Control of global instability in a non-parallel near wake, J. Fluid Mech. 404 (2000) 345-378.

[11] K.R. Sreenivasan, S. Raghu, D. Kyle, Absolute instability in variable density round jets, Exp. Fluids 7 (1989) 309-317.

[12] P.A. Monkewitz, D.W. Bechert, B. Barsikow, B. Lehmann, Self-excited oscillations and mixing in a heated round jet, J. Fluid Mech. 213 (1990) 611-639.

[13] D.M. Kyle, K.R. Sreenivasan, The instability and breakdown of a round variable-density jet, J. Fluid Mech. 249 (1993) 619-664.

[14] M.-H. Yu, P.A. Monkewitz, Oscillations in the near field of a heated two-dimensional jet, J. Fluid Mech. 255 (1993) 323-347.

[15] R.J. Lingwood, Absolute instability of the boundary layer on a rotating disk, J. Fluid Mech. 299 (1995) 17-33.

[16] R.J. Lingwood, An experimental study of absolute instability of the rotating-disk boundary-layer fow, J. Fluid Mech. 314 (1996) $373-405$.

[17] R.J. Lingwood, Absolute instability of the Ekman layer and related rotating flows, J. Fluid Mech. 331 (1997) 405-428.

[18] R.J. Lingwood, On the impulse response for swept boundary-layer flows, J. Fluid Mech. 344 (1997) 317-334.

[19] J.-M. Chomaz, Absolute and convective instabilities in nonlinear systems, Phys. Rev. Lett. 69 (1992) 1931-1934.

[20] A.N. Kolmogorov, I.G. Petrovsky, N.S. Piskunov, Investigation of a diffusion equation connected to the growth of materials, and application to a problem in biology, Bull. Univ. Moscow Ser. Int. Sec. A 1 (1937) 1-25.

[21] G. Dee, J. Langer, Propagating pattern selection, Phys. Rev. Lett. 50 (1983) 383-386.

[22] G. Dee, Dynamical properties of propagating front solutions of the amplitude equations, Physica D 15 (1985) 295-304.

[23] E. Ben-Jacob, H. Brand, G. Dee, L. Kramer, J.S. Langer, Pattern propagation in nonlinear dissipative systems, Physica D 14 (1985) 348-364.

[24] W. van Saarloos, Dynamical velocity selection: marginal stability, Phys. Rev. Lett. 58 (1987) 2571-2574.

[25] W. van Saarloos, Front propagation into unstable states: marginal stability as a dynamical mechanism for velocity selection, Phys. Rev. A 37 (1988) 211-229.

[26] W. van Saarloos, Front propagation into unstable states. II. Linear versus nonlinear marginal stability and rate of convergence, Phys. Rev. A 39 (1989) 6367-6390.

[27] J.A. Powell, A.C. Newell, C.K.R.T. Jones, Competition between generic and nongeneric fronts in envelope equations, Phys. Rev. A 44 (1991) 3636-3652.

[28] W. van Saarloos, P. Hohenberg, Fronts, pulses, sources and sinks in generalized complex Ginzburg-Landau equations, Physica D 56 (1992) 303-367.

[29] J.-M. Chomaz, A. Couairon, Propagating pattern selection and causality reconsidered, Phys. Rev. Lett. 84 (2000) 1910-1913.

[30] I. Delbende, J.-M. Chomaz, Nonlinear convective/absolute instabilities in parallel two-dimensional wakes, Phys. Fluids 10 (1998) 2724 2736.

[31] P.A. Monkewitz, L.N. Nguyen, Absolute instability in the near-wake of two-dimensional bluff bodies, J. Fluids Struct. 1 (1987) $165-184$.

[32] P.G. Drazin, W.H. Reid, Hydrodynamic Stability, Cambridge University Press, 1981.

[33] P. Huerre, P.A. Monkewitz, Absolute and convective instabilities in free shear layers, J. Fluid Mech. 159 (1985) 151-168.

[34] A. Couairon, J.-M. Chomaz, Global instabilities in fully nonlinear systems, Phys. Rev. Lett. 77 (1996) 4015-4018.

[35] A. Couairon, J.-M. Chomaz, Absolute and convective instabilities, front velocities and Global Modes in nonlinear systems, Physica D 108 (1997) 236-276.

[36] A. Couairon, J.-M. Chomaz, Pattern selection in the presence of a cross fow, Phys. Rev. Lett. 79 (1997) 2666-2669.

[37] A. Couairon, J.-M. Chomaz, Fully nonlinear Global Modes in slowly varying fows, Phys. Fluids 11 (1999) 3688-3703.

[38] S.M. Tobias, M.R.E. Proctor, E. Knobloch, The role of absolute instability in the solar dynamo, Astron. Astrophys. 318 (1997) $55-58$.

[39] S.M. Tobias, M.R.E. Proctor, E. Knobloch, Convective and absolute instabilities of fluid flows in finite geometry, Physica D 113 (1998) 43-72.

[40] J. Fineberg, V. Steinberg, Vortex-front propagation in Rayleigh-Bénard convection, Phys. Rev. Lett. 58 (1987) $1332-1335$.

[41] H.W. Müller, M. Lücke, M. Kamps, Convective patterns in horizontal flow, Europhys. Lett. 10 (1989) 451-454.

[42] H.W. Müller, M. Lücke, M. Kamps, Transversal convection patterns in horizontal shear flow, Phys. Rev. A 45 (1992) 3714-3726.

[43] G. Ahlers, D.S. Cannel, Vortex-front propagation in rotating Couette-Taylor flow, Phys. Rev. Lett. 50 (1983) $1583-1586$.

[44] P.B. Büchel, M. Lücke, D. Roth, R. Schmitz, Pattern selection in the absolutely unstable regime as a nonlinear eigenvalue problem: Taylor vortices in axial flow, Phys. Rev. E 53 (1996) 4764-4777.

[45] G. Triantafyllou, G. Karniadakis, Computational reducibility of unsteady viscous flows, Phys. Fluids A 2 (1990) 653-656.

[46] P.A. Monkewitz, The absolute and convective nature of instability in two-dimensional wakes at low Reynolds numbers, Phys. Fluids 31 (1988) 999-1006.

[47] J.M. Chomaz, Fully nonlinear dynamiques of parallel wakes, J. Fluid Mech. (2003), in press.

[48] A. Couairon, J.-M. Chomaz, Pushed global modes in weakly inhomogeneous subcritical flows, Physica D 158 (2001) 129-150.

[49] D. Worledge, E. Knobloch, S.M. Tobias, M.R.E. Proctor, Dynamo waves in semi-infinite and finite domains, Proc. Roy. Soc. London Ser. A 453 (1997) 119-143.

[50] J.-M. Chomaz, A. Couairon, Against the wind, Phys. Fluids 11 (1999) 2977-2983.

[51] A. Soward, Thin aspect ratio $\alpha \omega$-dynamos in galactic discs and stellar shells, in: Advances in Nonlinear Dynamos, in: M. Nunez Jimenez, A. Feeriz-Mas (Eds.), The Fluid Mechanics of Astrophysics and Geophysics, Taylor \& Francis, 2001. 
[52] B. Pier, P. Huerre, J.-M. Chomaz, A. Couairon, Steep nonlinear Global Modes in spatially developing media, Phys. Fluids 10 (1998) 2433-2435.

[53] B. Pier, P. Huerre, J.-M. Chomaz, Bifurcation to fully nonlinear synchronized structures in slowly varying media, Physica D 148 (2001) 49-96.

[54] D. Harris, A.P. Bassom, A.M. Soward, An inhomogeneous Landau equation with application to spherical Couette flow in the narrow gap limite, Physica D 137 (2000) 260-276.

[55] B. Pier, P. Huerre, Nonlinear self-sustained structures and fronts in spatially developing wake flows, J. Fluid Mech. 435 (2001) $145-174$.

[56] J.-M. Chomaz, A. Couairon, S. Julien, Absolute and convective nature of the Eckhaus and zigzag instability with throughflow, Phys. Fluids 11 (1999) 3369-3373.

[57] J.T. Stuart, On finite amplitude oscillations in laminar mixing layers, J. Fluid Mech. 29 (1967) 417-440.

[58] R.T. Pierrehumbert, S.E. Widnall, The two- and three-dimensional instabilities of a spatially periodic shear layer, J. Fluid Mech. 114 (1982) 59-82.

[59] P.G. Potylitsin, W.R. Peltier, Three-dimensional destabilization of Stuart vortices: the influence of rotation and elliptique, J. Fluid Mech. 387 (1999) 205-226.

[60] G. Broze, F. Hussain, Nonlinear dynamics of forced transitional jets: periodic and chaotic attractors, J. Fluid Mech. 263 (1994) 93-132.

[61] S. Narayanan, F. Hussain, Measurements of spatiotemporal dynamics in a forced plane mixing layer, J. Fluid Mech. 320 (1996) $71-115$.

[62] L. Kaiktsis, G.E. Karniadakis, S.A. Orszag, Unsteadiness and convective instabilities in two-dimensional flow over a backward-facing step, J. Fluid Mech. 321 (1996) 157-187.

[63] W. Koch, On the spatio-temporal stability of primary and secondary crossflow vortices in a three-dimensional boundary layer, J. Fluid Mech. 456 (2002) 85-111.

[64] L. Brandt, C. Cossu, J.M. Chomaz, P. Huerre, D.S. Henningson, On the convectively unstable nature of optimal streaks in boundary layers, J. Fluid Mech. 485 (2003) 221-242.

[65] B. Pier, Transition in the boundary layer over a rotating disk, J. Fluid Mech. (2003), submitted for publication.

[66] I. Delbende, J.-M. Chomaz, P. Huerre, Absolute and convective instabilities in the Batchelor vortex: a numerical study of the linear impulse response, J. Fluid Mech. 355 (1998) 229-254.

[67] P. Gondret, P. Ern, L. Meignin, M. Rabaud, Experimental evidence of a nonlinear transition from convective to absolute instability, Phys. Rev. Lett. 82 (1999) 11442-11445.

[68] A. Hanna, A. Saul, K. Showalter, Detailed studies of propagating fronts in the iodate oxydation of arsenous acid, J. Am. Chem. Soc. 104 (1982) 3838-3845.

[69] M. Högberg, D. Henningson, Secondary instability of cross-flow vortices in Falkner-Skan-Cooke boundary layers, J. Fluid Mech. 368 (1998) 339-357. 